\title{
Mechanisms behind altered pulsatile intracranial pressure in idiopathic normal pressure hydrocephalus: role of vascular pulsatility and systemic hemodynamic variables
}

\author{
Karen Brastad Evensen ${ }^{1,2} \cdot$ Per Kristian Eide ${ }^{1,3}$ (D) \\ Received: 16 March 2020 / Accepted: 19 May 2020 / Published online: 12 June 2020 \\ (C) The Author(s) 2020
}

\begin{abstract}
Background The dementia subtype idiopathic normal pressure hydrocephalus (iNPH) has unknown etiology, but one characteristic is elevated intracranial pressure (ICP) wave amplitudes in those individuals who respond with clinical improvement following cerebrospinal fluid (CSF) diversion. To explore the mechanisms behind altered ICP wave amplitudes, we correlated central aortic blood pressure (BP) and ICP waveform amplitudes (intracranial aortic amplitude correlation) and examined how this correlation relates to ICP wave amplitude levels and systemic hemodynamic parameters.

Methods The study included 29 patients with probable iNPH who underwent continuous multi-hour measurement of ICP, radial artery BP, and systemic hemodynamic parameters. The radial artery BP waveforms were used to estimate central aortic BP waveforms, and the intracranial aortic amplitude correlation was determined over consecutive 4-min periods.

Results The average intracranial aortic amplitude correlation was $0.28 \pm 0.16$ at the group level. In the majority of iNPH patients, the intracranial aortic amplitude correlation was low, while in about 1/5 patients, the correlation was rather high (average Pearson correlation coefficient $\geq 0.4$ ). The degree of correlation was hardly influenced by systemic hemodynamic parameters.

Conclusions In about $1 / 5 \mathrm{iNPH}$ patients of this study, the intracranial aortic amplitude correlation (IAAC $\mathrm{AORTIC}_{\text {) }}$ was rather high (average Pearson correlation coefficient $\geq 0.4$ ), suggesting that cerebrovascular factors to some extent may affect the ICP wave amplitudes in a subset of patients. However, in 14/19 (74\%) iNPH patients with elevated ICP wave amplitudes, the intracranial aortic amplitude correlation was low, indicating that the ICP pulse amplitude in most iNPH patients is independent of central vascular excitation, ergo it is modulated by local cerebrospinal physiology. In support of this assumption, the intracranial aortic amplitude correlation was not related to most systemic hemodynamic variables. An exception was found for a subgroup of the patients with high systemic vascular resistance, where there was a correlation.
\end{abstract}

Keywords ICP wave amplitudes · Central aortic waveforms · Idiopathic normal pressure hydrocephalus $\cdot$ Autoregulation

This article is part of the Topical Collection on Neurosurgery general

Per Kristian Eide

p.k.eide@medisin.uio.no

1 Department of Neurosurgery, Oslo University Hospital Rikshospitalet, P.O.Box 4950, Nydalen, 0424 Oslo, Norway

2 Department of Informatics, Faculty of Mathematics and Natural Sciences, University of Oslo, Oslo, Norway

3 Institute of Clinical Medicine, Faculty of Medicine, University of Oslo, Oslo, Norway

\section{Introduction}

Idiopathic normal pressure hydrocephalus (iNPH) is a subtype of dementia incorporating gait ataxia, urinary incontinence, and cerebrospinal fluid (CSF) circulation failure, but with an unknown cause. We have previously reported that the iNPH patients responding clinically to CSF diversion surgery (shunt surgery) typically present with elevated ICP wave amplitudes measured invasively $[7,8]$. While elevated ICP wave amplitudes characterize iNPH shunt responders, the underlying mechanisms remains unclear. For example, are the ICP wave 
amplitudes primarily related to vascular factors such as the blood pressure (BP) wave amplitudes or are the ICP wave amplitudes more influenced by extravascular factors?

In particular, the association between pulsatile arterial BP and ICP may be one factor behind the delayed paravascular clearance of CSF tracer observed in vivo in individuals with iNPH [6, 34]. In 2012, a brain-wide paravascular route for transport of fluids and solutes, denoted the glymphatic (glialymphatic) system, was described [15]. Convective forces created by the pressure gradients from the arterial pulsatile BP were hypothesized to represent the primary driving force behind the antegrade transport of fluid and solutes along the blood vessels [15, 16, 24]. Moreover, reduced arterial pulsations, such as seen in arterial hypertension, are associated with hampered paravascular solute transport [24]. With regard to iNPH, we have proposed that restricted arterial BP pulsatility related to abnormal pulsatile ICP may hamper the paravascular waste removal [6].

The present study addressed to which degree the pulsatile ICP that is created from the pulsatile arterial BP is affected by extra-cerebrovascular factors in iNPH patients. As a surrogate marker of arterial BP pulsatility within the cranial cavity, we utilize the central aortic BP waveforms, which are close to the scene both for hemodynamic events and the intracranial arterial pulsations. The concept described here is illustrated in Fig. 1. Using terms from system analysis, we consider the pulsatile central aortic BP our input signal and the pulsatile ICP our output signal. The unknown system consists of the vascular (blood vessel and flow) and extra-cerebrovascular factors (brain parenchyma and CSF) and will act as a physiological filter on the central aortic BP waves. The measured ICP waves are then the final result. This yields that the correlation between central aortic BP and ICP waveforms, here denoted Intracranial Aortic Amplitude Correlation (IAAC AORTIC $_{\text {AOR }}$, provides information about the impact of extra-cerebrovascular factors on the ICP waveform. A high degree of correlation would suggest a direct transfer of the central aortic BP waveform to the ICP waveform (i.e., extracerebrovascular compartment hardly affects the ICP waveform). If the abnormal ICP waveform in iNPH patients primarily is determined by vascular BP pulsatility, we would expect IAAC $_{\text {AORTIC }}$ to be increased and to be affected by systemic vascular variables and various patient characteristics. We would, however, not expect that surrogate markers of the

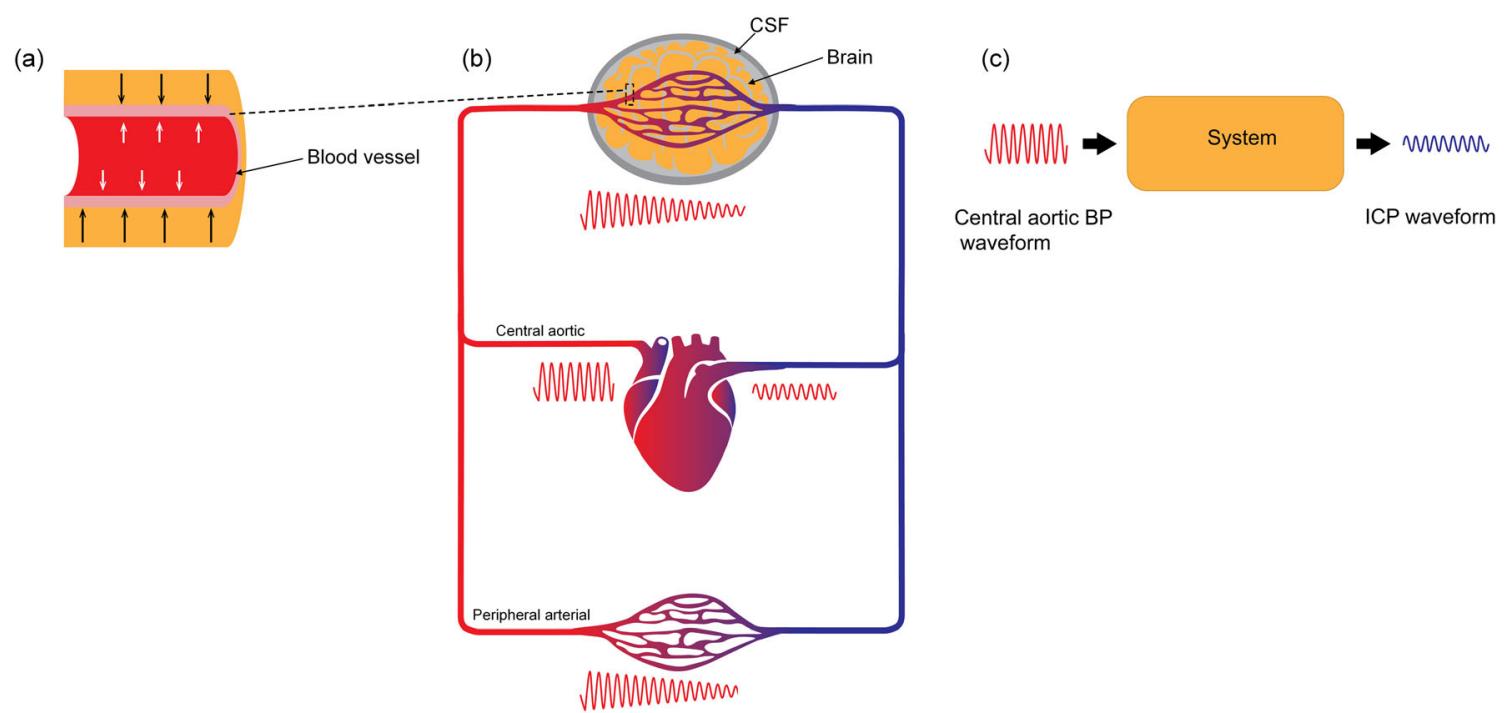

Fig. 1 The transmission from central aortic BP waveforms to ICP waveforms. a The pulsatile arterial BP causes pressure forces in the radial direction (white arrows) and towards the arterial blood vessel wall. The degree of movement of the arterial blood vessel wall partly depends on the properties of arterial wall (e.g., stiff blood pressure walls as seen in arterial hypertension, auto-regulatory capacity) and the blood flow (here collectively denoted vascular factor), and partly on the counter pressure (black arrows) determined by factors in the compartment outside the blood vessel (here denoted extra-cerebrovascular factor). b The cardiac contractions create arterial BP waveforms that propagate via the systemic circulation and cerebral circulation. Typically, the arterial $\mathrm{BP}$ waveform diminishes from the arterial (red) to the venous side (blue). While it is not feasible to continuously monitor the pulsatile arterial BP within the cranial compartment, the central aortic BP waveform is more closely related to the intracranial arterial $\mathrm{BP}$ waveform than peripheral arterial BP, e.g., the radial arterial BP waveform. c In this study, the "unknown" system consists of the vascular factors (intracranial blood vessels and cerebral blood flow) and extra-cerebrovascular factors (brain parenchyma and CSF). The central aortic BP waveform acts as a proxy for the intracranial arterial BP waveform and is our input signal, while the measured ICP waveform is the output signal. To study to which extent the vascular and extra-cerebrovascular factors constitute the system, we determined the Pearson correlation coefficient between amplitudes of central aortic and ICP waveforms for every $4 \mathrm{~min}$ period (referred to as Intracranial Aortic Amplitude Correlation, IAAC $\mathrm{AORTIC}_{\mathrm{C}}$ ). A high degree of correlation between input and output suggests a semidirect transfer from input to output, i.e., ICP is mainly determined by vascular factors (arterial BP) and the extra-cerebrovascular factors of the system are negligible. A low correlation indicates that the extracerebrovascular factors of the system highly affect the measured ICP signal. Illustration: Ine Eriksen, University of Oslo 
extra-cerebrovascular content to affect the correlation. Our hypothesis is, therefore, that the transfer of central aortic BP to ICP waveform primarily is affected by extracerebrovascular factors and that the elevated pulsatile ICP seen in iNPH patients are due to other pathophysiological factors than vascular factors per se.

\section{Materials and methods}

\section{Patients}

The study included patients with probable iNPH [33] who underwent work-out for CSF diversion surgery at the Department of Neurosurgery, Oslo University HospitalRikshospitalet, from October 2008 to January 2009. Multihour continuous ICP monitoring with the determination of mean ICP wave amplitude ( $\mathrm{MWA}_{\mathrm{ICP}}$ ) is part of the clinical routine and an important factor when deciding on whether to perform CSF diversion surgery. Individuals with mean ICP wave amplitudes $\left(\mathrm{MWA}_{\mathrm{ICP}}\right)$ above a selected threshold are offered shunt surgery, as previously described [7].

\section{Monitoring and analysis of continuous pressure and hemodynamic variables}

The ICP was measured continuously using a solid ICP sensor (Codman MicroSensor ${ }^{\mathrm{TM}}$, Johnson \& Johnson, Raynham, MA, USA) that had been placed $1-2 \mathrm{~cm}$ into the frontal brain parenchyma through a small burr hole and a minimal opening in the dura in local anesthesia. The radial artery BP was measured continuously and invasively from the right radial artery using a Truwave PX-600F Pressure Monitoring Set (Edwards Life sciences LLC, Irvine, CA) that was placed at the level of the heart. Both the ICP waveform and radial artery BP waveforms were sampled at $200 \mathrm{~Hz}$, which is an adequate sampling rate [14] and digitized using an analogue-to-digital converter (Sensometrics ${ }^{\circledR}$ Pressure Logger; dPCom AS, Oslo, Norway) before they were stored as raw data files with an identical time reference. The continuous pressure signals were analyzed using Sensometrics $\circledR^{\circledR}$ software (dPCom AS, Oslo, Norway).

Central aortic BP waveforms were estimated from the radial artery BP waveforms using the SphygmoCor system (SphygmoCor®; AtCor Medical, West Ryde, NSW, Australia). The SphygmoCor system has been validated to successfully estimate central aortic BP waveforms from several different variants of peripheral BP measurements. The most relevant validation studies are brachial artery BP measurements [17], invasive radial artery BP measurements [25], and radial artery tonometry measurements [3, 12]. In addition, the SphygmoCor system has been approved for clinical use by regulatory bodies such as the US FDA [12].
Systemic hemodynamic variables were measured simultaneously with the continuous pressure signals using the LiDCO ${ }^{\mathrm{TM}}$ plus software (version 4.0, LiDCO Ltd., Cambridge, UK), which provides for a minimally invasive technique of hemodynamic monitoring. The methodology incorporates two methods, namely, a continuous arterial BP waveform analysis system (PulseCO), coupled to a singlepoint lithium indicator dilution calibration system (LiDCO) $[26,29]$. The calibration procedure incorporates an injection of $0.3 \mathrm{mmol}$ lithium chloride through a peripheral line [29]. The lithium is detected by an external lithium ion-sensitive external electrode connected to the peripheral arterial line, which enables monitoring of the hemodynamic variables cardiac output (CO), systemic vascular resistance (SVR), stroke volume (SV), cardiac index (CI), mean radial arterial BP, and heart rate (HR). The software in LiDCO ${ }^{\mathrm{TM}}$ plus and Sensometrics ${ }^{\circledR}$ has an identical time reference. We used a minimally invasive approach to monitor the systemic hemodynamic variables, namely, the PulseCO hemodynamic monitor. This approach has previously been validated against the pulmonary artery catheter method [19] and applied for hemodynamic monitoring in several patient cohorts $[2,20]$.

In this study, for the first time, the moving correlation between single-pressure wave amplitudes of ICP and central aortic BP waveform amplitudes were determined (Intracranial Aortic Amplitude Correlation; IAAC AORTIC $_{\text {) }}$. For this purpose, the peak to peak amplitudes of corresponding central aortic BP and ICP single pressure waves were automatically identified. In the following, the peak to peak amplitude refers to the difference between the maximum systolic BP and the minimum diastolic BP for the central aortic $\mathrm{BP}$ single waves and the difference between the maximum ICP and minimum ICP for the ICP single waves. The amplitude identification did not identify the $\mathrm{P} 1, \mathrm{P} 2$, and $\mathrm{P} 3$ peaks, but identified the highest peak between two diastolic minimum pressures. The intracranial aortic amplitude correlation (IAAC $\left.\mathrm{AORTIC}_{\mathrm{A}}\right)$ was thereafter determined as Pearson correlation coefficient for every consecutive 4-min period of the individual central aortic BP and ICP recording (Fig. 2). In previous studies, we have used a similar approach based on arterial BP measurements from the radial artery (intracranial arterial amplitude correlation; IAAC) $[4,10]$.

In the present study, we further explored the degree of intracranial aortic amplitude correlation for various levels of mean ICP wave amplitude ( $\left.\mathrm{MWA}_{\mathrm{ICP}}\right)$, systemic hemodynamic variables, and how the correlation related to various patient characteristics and to clinical response to CSF diversion surgery.

\section{Time alignment of pressure signals}

As a central part of the study is to examine the correlation between the amplitudes of single waves resulting from the 

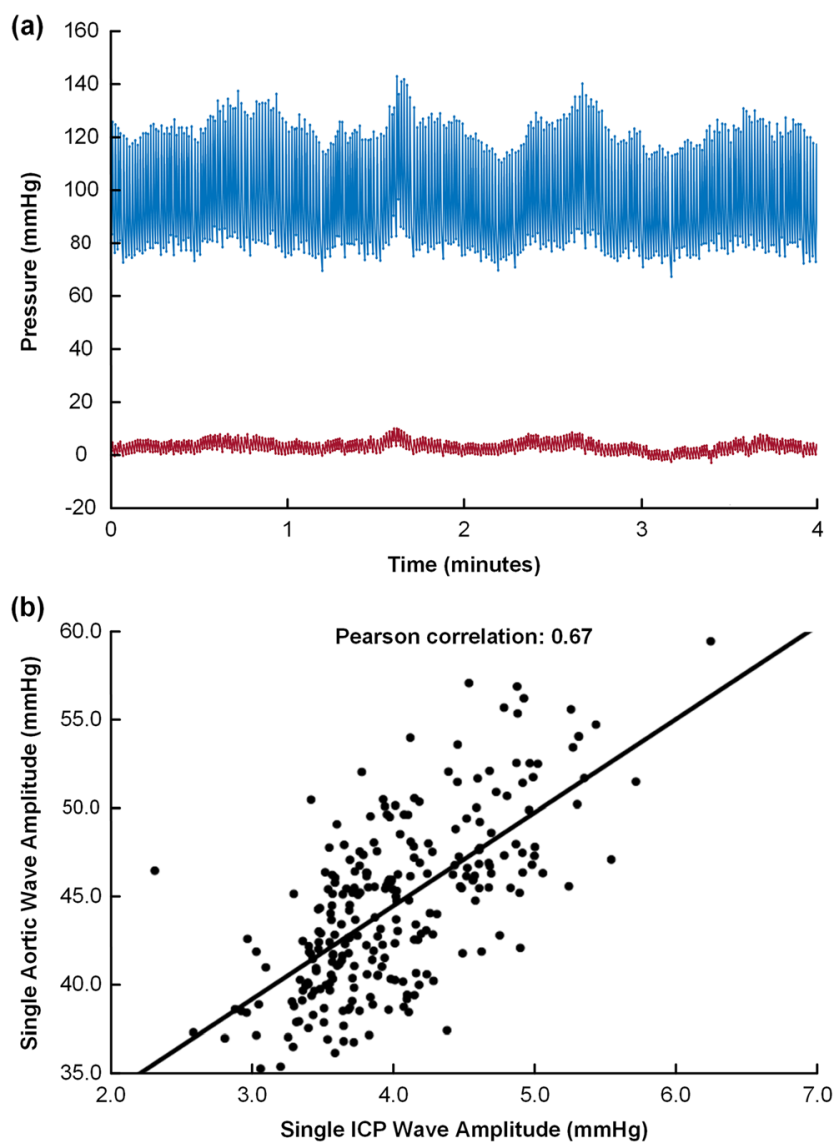

Fig. 2 The intracranial aortic amplitude correlation. a Simultaneous central aortic blood pressure (BP; blue waveform) and intracranial pressure (ICP) single-pressure waves (red waveform) were automatically identified and the single aortic BP and ICP wave amplitude values determined for every aortic BP/ICP single wave pair. b The Pearson correlation coefficient of corresponding aortic BP/ICP single-wave amplitudes (intracranial aortic amplitude correlation, IAAC $_{\text {AORTIC }}$ ) was determined for consecutive 4-min period. In this example, the Pearson correlation coefficient was $0.67(P<0.001)$. Notably, this example refers to one particular 4-min period; the magnitude of this IAAC $_{\text {AORTIC }}$ observation was substantially higher than observed in the vast majority of 4-min periods

same heartbeat, perfect time alignment between the signals was crucial. To exclude uncertainties, time alignment of the time series was thoroughly checked, initially by visual comparison. If apparent artifacts due to patient movement did not align, or the time delay between signals shifted throughout the recording, there was reason to believe that there had been some corruption of the signal. In the dataset presented here, there was a systematically recurring time shift that only happened in one of the time series (central aortic BP waveforms). The time shift was corrected using a two-step approach. First, the onset of each single wave was calculated by a beat detection algorithm that utilizes a curve length transformation [38]. The onset before the anomaly was chosen for both time series and joined with the time aligned onset $6 \mathrm{~s}$ after, thereby removing the anomaly. The diastolic pressure over such a short timeframe was found to be approximately constant.

\section{Statistics}

The statistical analyses were performed using the SPSS software version 25 (IBM Corporation, Armonk, NY). Differences between continuous data were determined using one-way ANOVA and Bonferroni post hoc tests for multiple comparisons. Statistical significance was accepted at the 0.05 level.

\section{Results \\ Patients}

The study included 29 patients with probable iNPH; demographic and management data are presented in Table 1. Twenty-two patients were shunted and seven managed conservatively. All iNPH patients had normal static ICP (mean ICP) $<18 \mathrm{mmHg}$, which is required for the diagnosis probable iNPH [33]. The iNPH patients responding clinically to CSF diversion surgery had elevated mean ICP wave amplitudes $\left(n=20 ; \mathrm{MWA}_{\mathrm{ICP}}=5.8 \mathrm{mmHg}\right)$, as compared with the nine iNPH patients managed conservatively or without clinical response to surgery $\left(\mathrm{MWA}_{\mathrm{ICP}}=3.5 \mathrm{mmHg} ; P<0.001\right.$; independent samples $t$ test).

\section{The intracranial aortic amplitude correlation for different levels of mean ICP wave amplitude}

The individual average levels of mean ICP wave amplitude $\left(\mathrm{MWA}_{\mathrm{ICP}}\right)$, determined from observations every $6 \mathrm{~s}$, are presented in Table 2. With reference to our established thresholds [7], $\mathrm{MWA}_{\text {ICP }}$ was above threshold $(>4.0 \mathrm{mmHg})$ in $19 / 29$ $(66 \%)$ individuals. At the group level, the average $\mathrm{MWA}_{\mathrm{ICP}}$ was $5.0 \pm 1.8 \mathrm{mmHg}$ (Table 2).

The individual average values for intracranial aortic amplitude correlation $\left(\mathrm{IAAC}_{\mathrm{AORTIC}}\right.$ ) over consecutive 4-min periods are shown in Table 2. The average number of 4-min periods available for analysis for individuals of this cohort was $254 \pm$ 64 . At the group level, the average intracranial aortic amplitude correlation (IAAC AORTIC $_{\text {) was }} 0.28 \pm 0.16$ (Table 2). While a threshold level for IAAC AORTIC $_{\text {A }}$ has previously not been established, given a threshold of IAAC $_{\text {AORTIC }} \geq 0.4,6 / 29$ (21\%) of individuals were above threshold.

Among the 19 individuals with $\mathrm{MWA}_{\mathrm{ICP}} \geq 4.0 \mathrm{mmHg}$, IAAC $_{\text {AORTIC }} \leq 0.4$ was observed in $14(74 \%)$ (Table 3 ). Moreover, among the 10 patients with $\mathrm{MWA}_{\mathrm{ICP}}<$ $4.0 \mathrm{mmHg}$, one individual $(10 \%)$ had IAAC $_{\text {AORTIC }} \geq 0.4$. Even though $\mathrm{MWA}_{\mathrm{ICP}}$ tended to be higher when IAAC $_{\text {AORTIC }}$ increased, this was not significant $(P=0.30$; Pearson chi-square test). Defining a threshold of IAAC $_{\text {AORTIC }}$ of 0.3 gave no different results $(P=0.68$; Pearson chi-square test). We also determined the association 
Table 1 Material of patients with probable iNPH

\begin{tabular}{ll}
\hline iNPH patients $(N)$ & 29 \\
Age (years) & $75 \pm 6$ years \\
Gender $(\mathrm{F} / \mathrm{M})$ & $15 / 14$ \\
$\mathrm{BMI}\left(\mathrm{kg} / \mathrm{m}^{2}\right)$ & $24.1 \pm 3.7$ \\
Co-morbidity with arterial hypertension and/or diabetes mellitus $(N)$ & 11 \\
Symptom duration (years) & $2.5 \pm 2.2$ \\
Severity of iNPH symptoms (iNPH grading scale ${ }^{\mathrm{a}}$; median, ranges) & $10(7,13)$ \\
Treatment & $22 / 7$ \\
$\quad$ CSF diversion surgery/no surgery $(N)$ & \\
Response to CSF diversion surgery & 20 \\
Positive clinical response $\left(\right.$ Change in an iNPH grading scale $\left.{ }^{\mathrm{a}}>1 ; N\right)$ & 2 \\
$\quad$ No positive clinical response $(N)$ &
\end{tabular}

${ }^{a}$ iNPH grading scale, ref. Eide and Sorteberg, 2010. Continuous data presented as average \pm standard deviation

Table 2 Individual mean ICP wave amplitude $\left(\mathrm{MWA}_{\mathrm{ICP}}\right)$ vs. intracranial aortic amplitude correlation (IAAC $\mathrm{AORTIC}_{\text {) }}$

\begin{tabular}{|c|c|c|c|c|c|c|c|c|}
\hline \multirow[t]{2}{*}{ Patient } & \multicolumn{4}{|c|}{ Mean ICP wave amplitude $\left(\mathrm{MWA}_{\mathrm{ICP}}\right)$} & \multicolumn{4}{|c|}{ Intracranial aortic amplitude correlation (IAAC AORTIC $_{\text {) }}$} \\
\hline & 6-s observations $(N)$ & Average $(\mathrm{mmHg})$ & $\geq 4.0 \mathrm{mmHg}$ & $<4.0 \mathrm{mmHg}$ & 4-min observations $(\mathrm{N})$ & Average & $\geq 0.4$ & $<0.4$ \\
\hline 1 & 6764 & 3.2 & & 1 & 103 & 0.33 & & 1 \\
\hline 2 & 7819 & 6.9 & 1 & & 350 & 0.12 & & 1 \\
\hline 3 & 8114 & 3.3 & & 1 & 325 & 0.47 & 1 & \\
\hline 4 & 7532 & 5.1 & 1 & & 325 & 0.32 & & 1 \\
\hline 5 & 4810 & 5.6 & 1 & & 189 & 0.22 & & 1 \\
\hline 6 & 5280 & 5.5 & 1 & & 238 & 0.40 & 1 & \\
\hline 7 & 7226 & 5.0 & 1 & & 119 & 0.29 & & 1 \\
\hline 8 & 4610 & 3.9 & & 1 & 237 & 0.31 & & 1 \\
\hline 9 & 4757 & 3.6 & & 1 & 237 & 0.25 & & 1 \\
\hline 10 & 6721 & 9.4 & 1 & & 346 & 0.66 & 1 & \\
\hline 11 & 6343 & 4.1 & 1 & & 326 & 0.21 & & 1 \\
\hline 12 & 5762 & 3.0 & & 1 & 233 & 0.03 & & 1 \\
\hline 13 & 7508 & 7.4 & 1 & & 325 & 0.55 & 1 & \\
\hline 14 & 5191 & 4.9 & 1 & & 236 & 0.18 & & 1 \\
\hline 15 & 3933 & 3.9 & & 1 & 194 & 0.03 & & 1 \\
\hline 16 & 4892 & 4.8 & 1 & & 301 & 0.12 & & 1 \\
\hline 17 & 5704 & 3.6 & & 1 & 264 & 0.37 & & 1 \\
\hline 18 & 4021 & 4.4 & 1 & & 208 & 0.10 & & 1 \\
\hline 19 & 8682 & 4.1 & 1 & & 234 & 0.12 & & 1 \\
\hline 20 & 5681 & 10.9 & 1 & & 267 & 0.16 & & 1 \\
\hline 21 & 4918 & 5.6 & 1 & & 222 & 0.59 & 1 & \\
\hline 22 & 4172 & 3.9 & & 1 & 260 & 0.34 & & 1 \\
\hline 23 & 4136 & 6.2 & 1 & & 223 & 0.15 & & 1 \\
\hline 24 & 8161 & 5.3 & 1 & & 357 & 0.30 & & 1 \\
\hline 25 & 8086 & 4.6 & 1 & & 327 & 0.44 & 1 & \\
\hline 26 & 6350 & 3.3 & & 1 & 207 & 0.22 & & 1 \\
\hline 27 & 6492 & 6.2 & 1 & & 244 & 0.21 & & 1 \\
\hline 28 & 5506 & 2.9 & & 1 & 255 & 0.17 & & 1 \\
\hline 29 & 4487 & 5.5 & 1 & & 208 & 0.39 & & 1 \\
\hline $\mathrm{AVG} \pm \mathrm{STDEV}$ & $5988 \pm 1439$ & $5.0 \pm 1.8$ & & & $254 \pm 64$ & $0.28 \pm 0.16$ & & \\
\hline$N$ & & & 19 & 10 & & & 6 & 23 \\
\hline
\end{tabular}


Table 3 Number of individuals with $\mathrm{MWA}_{\mathrm{ICP}} / \mathrm{IAAC}_{\mathrm{AORTIC}}$ combinations above or below thresholds

\begin{tabular}{llll}
\hline & \multicolumn{2}{l}{ MWA $_{\text {ICP }}$ (mean ICP wave amplitude) } \\
\cline { 2 - 3 } & & $\geq 4.0 \mathrm{mmHg}$ & $<4.0 \mathrm{mmHg}$ \\
\hline IAAC $_{\text {AORTIC }}$ (intracranial aortic amplitude correlation) & $>0.4$ & 5 & 1 \\
& $<0.4$ & 14 & 9 \\
\hline
\end{tabular}

$P=0.30$ (Pearson chi-square test)

between individual average levels of IAAC $_{\text {AORTIC }}$ and MWA $_{\text {ICP }}$ that was non-significant (Fig. 3).

\section{The intracranial aortic amplitude correlation versus systemic hemodynamic variables}

The intracranial aortic amplitude correlations (IAAC $\mathrm{AORTIC}_{\text {) }}$ present in Table 2 were assessed for various levels of the systemically measured hemodynamic variables. Since the IAAC $_{\text {AORTIC }}$ is an index derived from a correlation coefficient, we decided to assess categories of the systemic hemodynamic variables. The correlation IAAC $_{\text {AORTIC }}$ was significantly higher in the group with systemic vascular resistance (SVR) above 1600 , compared to the group with SVR in the range $1200-1600$ dynes $/ \mathrm{s} / \mathrm{cm}^{5}$. However, the degree of correlation was not different for other levels of the systemic hemodynamic variables. Figure 4 presents average values of IAAC $_{\mathrm{AORTIC}}$ for various levels of CO (Fig. 4a), SVR (Fig. 4b), SV (Fig. 4c), CI (Fig. 4d), mean arterial BP (Fig. 4e), and HR (Fig. 4f).

\section{The intracranial aortic amplitude correlation versus patient characteristics}

It could be expected that patient characteristics substantially modify the intracranial aortic amplitude correlation. However, as shown in Fig. 5, we found no difference in IAAC $_{\text {AORTIC }}$ for various categories of age (Fig. 5a), BMI (Fig. 5b), duration of

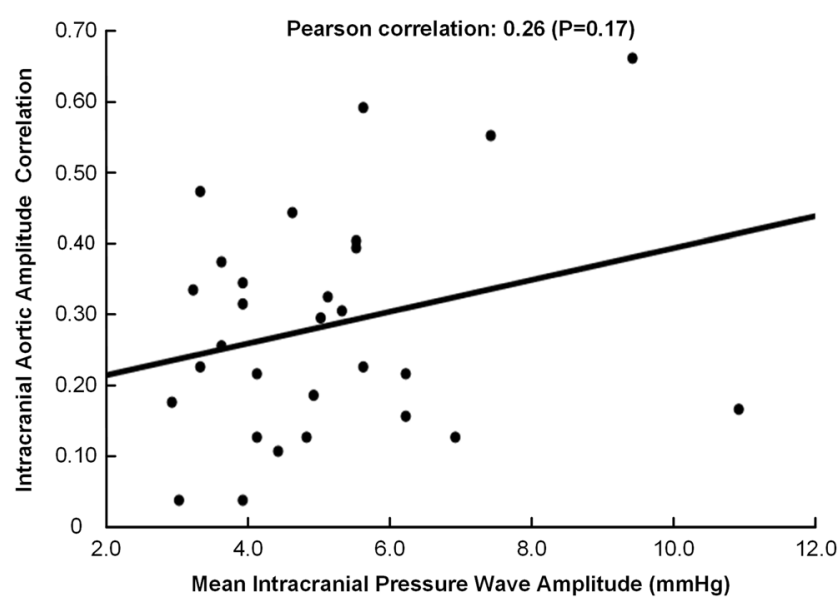

Fig. 3 Association between intracranial aortic amplitude correlation and the level of mean ICP wave amplitude. The IAAC $_{\text {AORTIC }}$ is plotted for different levels of $\mathrm{MWA}_{\mathrm{ICP}}$, including presentation of the fit line and the Pearson correlation coefficient (R) with $P$ value disease (Fig. 5c), or presence of co-morbidity (i.e., arterial hypertension and/or diabetes mellitus; Fig. 5d). Another point worth investigating is whether IAAC $_{\text {AORTIC }}$ is dependent on the clinical response to CSF diversion (shunt) surgery. We found no evidence that neither shunt response (Fig. 6a) nor the degree of shunt response (Fig. 6b) was related to the level of IAAC AORTIC $_{\text {in this study. }}$

\section{Discussion} between intracranial and aortic pressure amplitudes at the

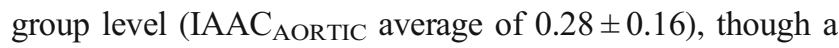
higher degree of correlation $(\geq 0.4)$ was seen in about $1 / 5$ iNPH patients. Moreover, the correlation was hardly affected by systemic hemodynamic variables, except for in a subgroup with increased systemic vascular resistance.

The presently reported elevated mean ICP wave amplitudes in iNPH patients responding favorably to CSF diversion surgery confirm previous clinical experience from larger iNPH patient cohorts $[7,8]$.

In the past, the relationship between radial arterial BP and ICP pressure signals has been studied extensively in the frequency domain and been referred to as transfer function or systems analysis $[27,28]$. These studies were interpreted to provide evidence that loss of vasomotor tone of the precapillary vessels changed the radial arterial BP to ICP transmission into a passive and linear pressure transmission [28, 30,31]. As loss of vasomotor tone is an indication of impaired autoregulation, this implies that the physical mechanisms that dampen parts of the frequency spectrum are reduced or diminished when autoregulation is reduced. As a result, the correlation between mean radial arterial BP and mean ICP was established as a surrogate marker of intracranial pressure autoregulation [36]. When autoregulation is impaired, the physical mechanisms dampen less, and the correlation increases. While comparing mean levels, the correlation is denoted the cerebrovascular Pressure-Reactivity index (PRx). A similar index exists for the correlation between single-pressure wave amplitudes of ICP and radial arterial BP $[1,11]$.

The current study explored a comparable concept to that explained in the previous work $[27,28]$, but differs from previous studies by utilizing the central aortic BP waveform for the first time. A limitation with the studies utilizing radial
The main observation was a rather low degree of correlation 
Fig. 4 The intracranial aortic amplitude correlation for different categories of systemic hemodynamic parameters. The level of intracranial aortic amplitude correlation (IAAC ${ }_{\text {AORTIC }}$ is presented for different categories of a cardiac output, b systemic vascular resistance, $\mathbf{c}$ stroke volume, $\mathbf{d}$ cardiac index, e mean arterial blood pressure, and $\mathbf{f}$ heart rate. Each error bar is presented as mean with 95\% CI. Except for significantly different

IAAC $_{\text {AORTIC }}$ between the categories with systemic vascular resistance $1200-1600$ or $>1600$ dynes $/ \mathrm{s} / \mathrm{cm}^{5}$ (b), there were no significant differences between groups (ANOVA with post hoc Bonferroni tests) (a)
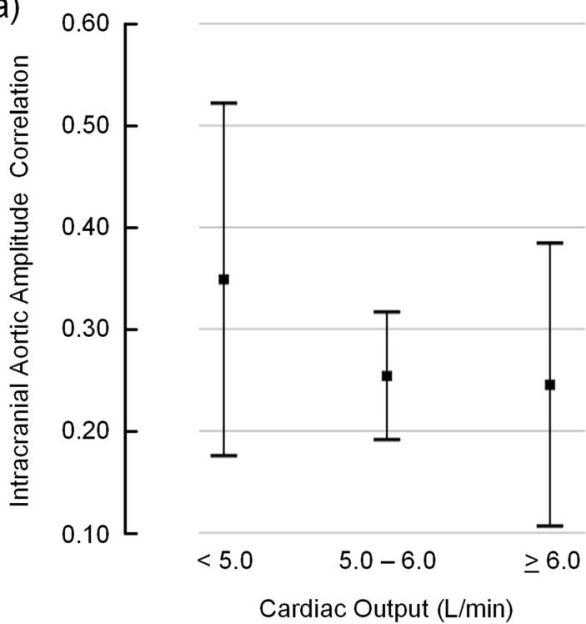

(b)
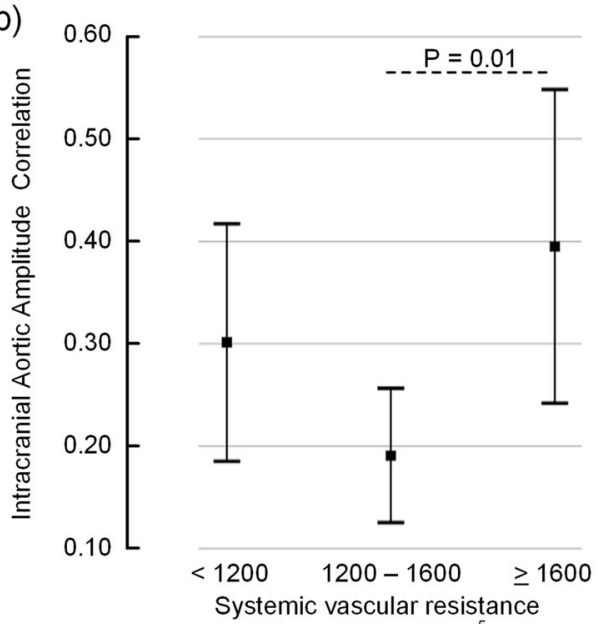

(c)

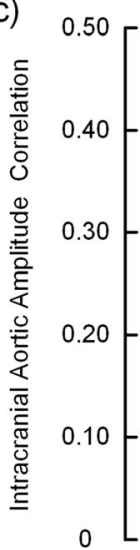

(d)

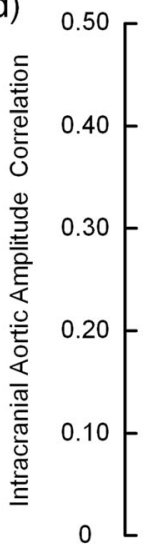

(e)
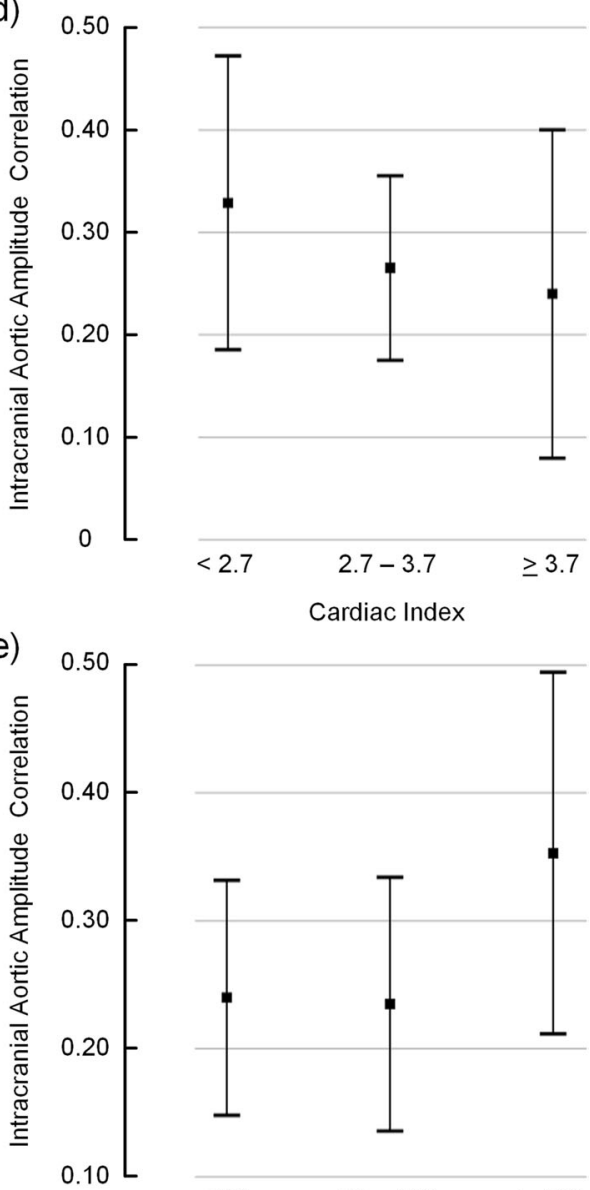

Cardiac Index

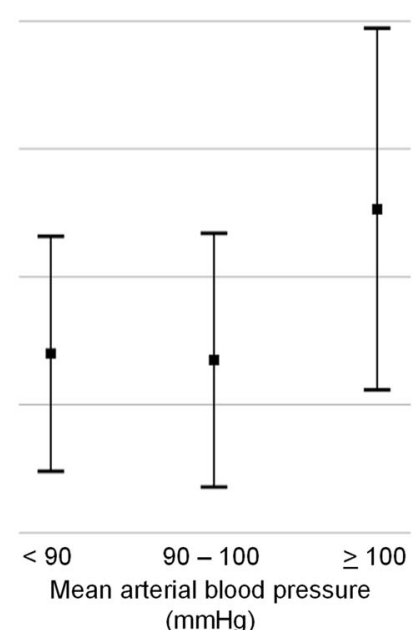

(f)

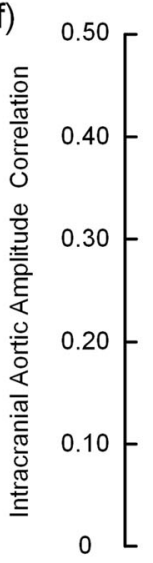

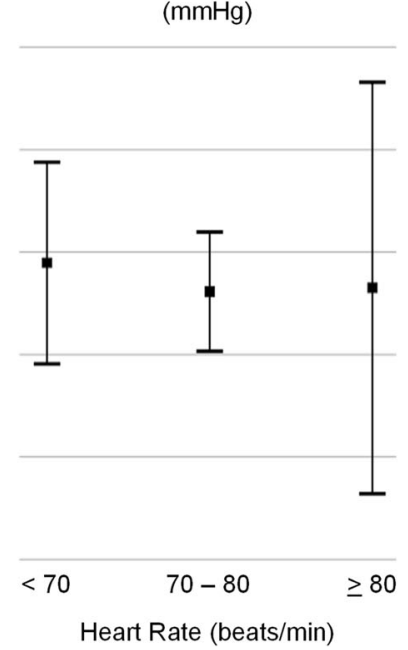

Stroke volume $(\mathrm{mL} /$ beat $)$

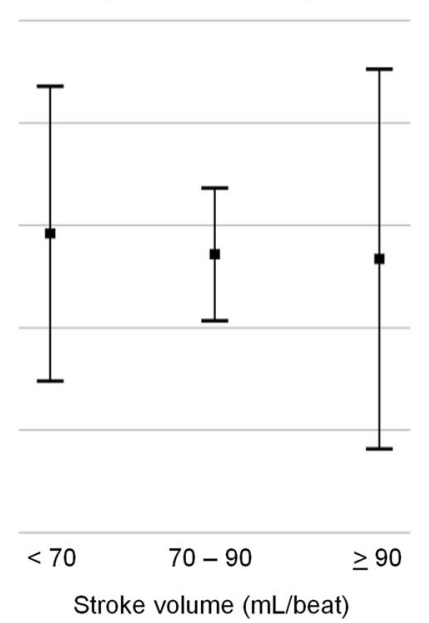

artery BP measurements for IAAC estimation $[4,10,11]$ is that the radial artery is more peripheral to the brain than the aortic artery. A criticism against use of radial artery measurements is that the BP measurements are too far from the intracranial compartment. In this regard, we would expect a closer association between central aortic BP waveforms and ICP waveforms making central aortic BP estimates more relevant.
While previous studies have primarily addressed the role of cerebrovascular factors on the ICP waveform, we here aimed at focusing on both the cerebrovascular and the extracerebrovascular factors. The latter causes physical filtering of the intracranial arterial BP waveform (see Fig. 1). Using the wave amplitude as the primary waveform characteristic, we investigated the role played by the source (the arterial BP 
Fig. 5 The intracranial aortic amplitude correlation for different categories of patient characteristics. The level of intracranial aortic amplitude correlation (IAAC $\mathrm{AORTIC}_{\mathrm{C}}$ ) is presented for different categories of patient data, including a age, $\mathbf{b}$ body mass index (BMI), c duration of disease, and $\mathbf{d}$ presence of co-morbidity (i.e., arterial hypertension and/or diabetes mellitus). Each error bar is presented as mean with $95 \%$ CI. There were no significant differences between groups (ANOVA with post hoc Bonferroni tests) (a)
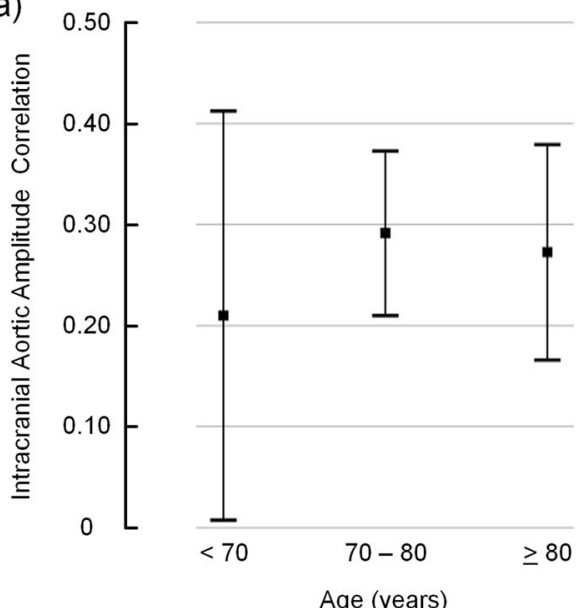

(b)

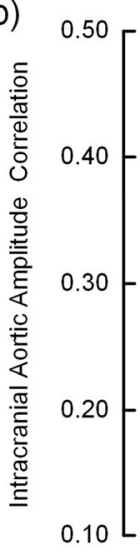

(c)

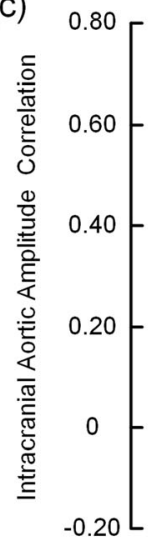

(d)

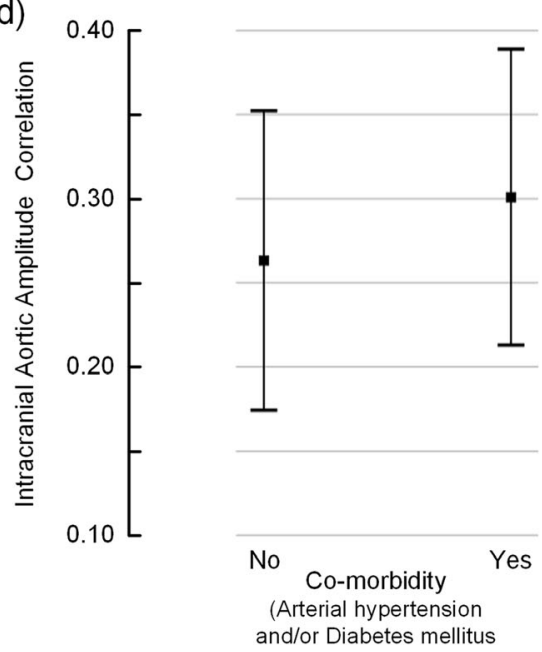

waveform) and the filter (the intracranial constituents). Intracranial aortic amplitude correlation values approaching 0 indicate low degree of association, indicating that the ICP waveform is less impacted by vascular factors (preserved autoregulation) and that is primarily determined by extracerebrovascular factors (i.e., alterations in the brain and $\mathrm{CSF}$ ). Correlation values approaching +1 , on the other hand, implies a direct association between alterations in arterial BP and ICP, which suggest a more extensive role of vascular factors such as impaired pressure autoregulation and altered cerebral blood flow.

There are presently no established threshold values for which intracranial aortic amplitude correlation values represent an upper threshold value. We would expect this correlation to be higher than previously reported correlation levels that were based on peripheral arterial BP measurements. In comparison, despite the general agreement that the traditional PRx and IAAC indices of cerebrovascular pressure-reactivity can be looked upon as surrogate markers of intracranial pressure autoregulation, the threshold levels for impairment/not impairment have not been defined. A clinical study showed that the outcome seems to worsen when PRx remained above
0.2-0.3 [39] among a cohort of individuals with traumatic brain injury. A different study reported that average values of amplitude correlation above 0.2 during week 1 after a subarachnoid hemorrhage was associated with worse outcome [11]. The thresholds for impaired autocorrelation are thereby clearly in the lower part of the spectrum.

In the present study, the average correlation between ICP and central aortic BP amplitudes was low in our cohort $\left(\mathrm{IAAC}_{\mathrm{AORTIC}} 0.28 \pm 0.16\right)$ and hardly influenced by the systemic hemodynamic variables. However, in $6 / 29$ of the patients $(21 \%)$, the average correlation IAAC $_{\text {AORTIC }}$ was above 0.40 . This might indicate that cerebrovascular factors play a dominating role in determining the ICP wave amplitude level in this subgroup. The cerebrovascular factors may be impaired cerebral pressure autoregulation as well as cerebrovascular disease that together affect cerebral blood flow and thereby the ICP wave amplitudes. Cardiovascular risk factors are more prevalent in $\mathrm{iNPH}$; the prevalence of arterial hypertension and diabetes is increased in patients with iNPH [5]. This fits well with our findings of increased correlation IAAC $_{\text {AORTIC }}$ for a subgroup with high systemic vascular resistance. Another aspect is that BP waveforms change with age. Notably, iNPH is a disease of the 
(a)

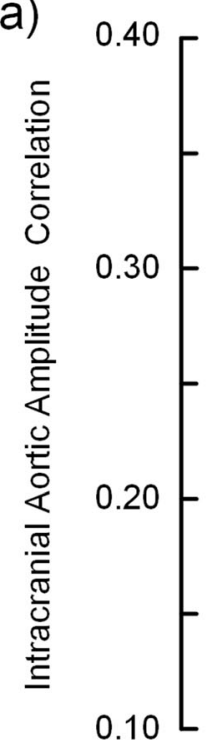

(b)

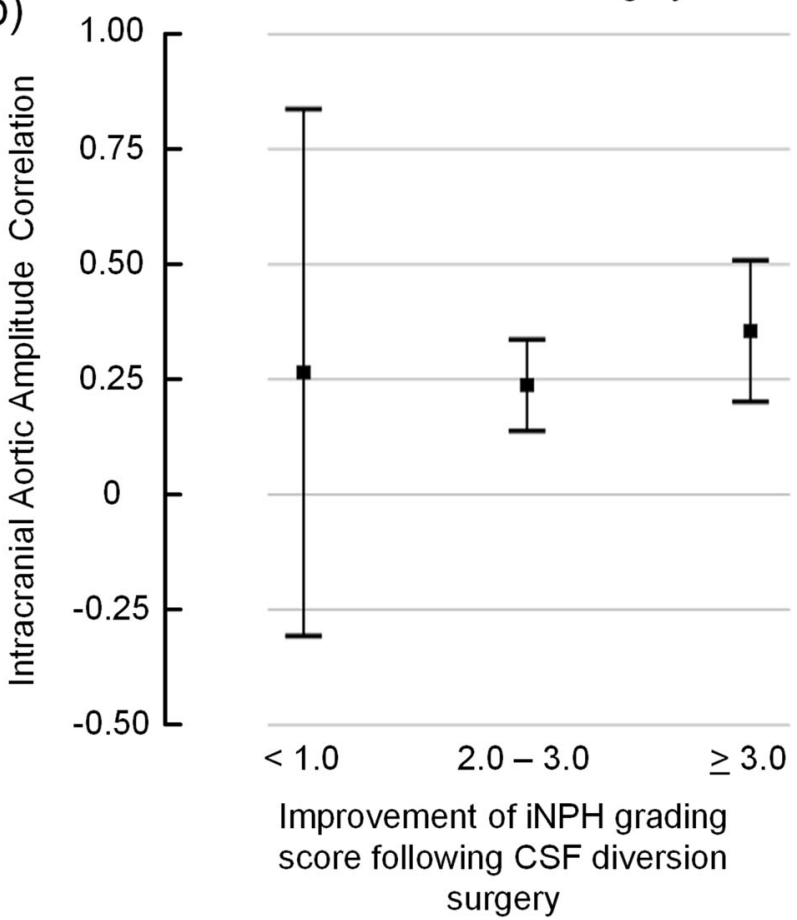

Fig. 6 The intracranial aortic amplitude correlation for different categories of clinical response to CSF diversion surgery. The level of intracranial aortic amplitude correlation (IAAC $\mathrm{AORTIC}_{\mathrm{C}}$ ) is presented for a the sub-groups of individuals with either no positive clinical response or a positive clinical response to CSF diversion surgery, and $\mathbf{b}$ for subgroups with different degree of clinical response to CSF diversion surgery. Each error bar is presented as mean with $95 \%$ CI. There were no significant differences between groups (ANOVA with post hoc Bonferroni tests, and revealed)

elderly. Elevated ICP wave amplitudes in these individuals could be an age phenomenon. In line with this assumption, a study by Lloyd et al. [22] reported an age-dependent change in the intracranial arterial waveform that corresponded well with the arterial wall stiffening seen with increased age. However, we

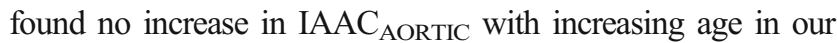
cohort. If the intracranial arterial $\mathrm{BP}$ waveform was a decisive factor for pulsatile ICP, we would, therefore, expect an agedependent $\mathrm{IAAC}_{\mathrm{AORTIC}}$, as the amplitude is the major waveform characteristic.

Among the present 19 patients with ICP wave amplitudes above the threshold (MWA $\mathrm{ICP} \geq 4.0 \mathrm{mmHg}$ ), 5/19 individuals $(26 \%)$ also presented with IAAC ${ }_{\text {AORTIC }}$ indices above 0.4 . According to our model, the levels of ICP wave amplitudes in this subgroup might be partly affected by cerebrovascular factors such as impaired autoregulation. However, in the majority of the patients with iNPH, the levels of ICP wave amplitudes seemed to be primarily determined by extracerebrovascular factors. Accordingly, in $74 \%$ of iNPH individuals, the increased ICP wave amplitudes were accompanied with IAAC $_{\text {AORTIC }}$ below 0.4. Moreover, at the group level, the intracranial aortic amplitude correlation was not different for various levels of mean ICP wave amplitude (Fig. 3). On this background, we find it difficult to explain elevated ICP wave amplitudes by altered intracranial arterial BP amplitudes and suggest it is necessary to look at other possible causes of elevated pulsatile ICP in the majority of iNPH patients. In this regard, the recently described glymphatic system for transport of fluid and solutes in the central nervous system [15] could be particularly relevant. The glymphatic system may play a critical role in the brain's ability to remove toxic metabolic waste products [32], and glymphatic magnetic resonance imaging (gMRI) gave evidence of impaired glymphatic function in iNPH patients $[6,34]$. In rodents, reduced arterial pulsations, such as seen in arterial hypertension, were associated with hampered antegrade transport of fluid and solutes along the blood vessels [24]. Likewise, we hypothesize that unfavorable properties of the extracerebrovascular compartment may cause restriction of intracranial arterial BP pulsations and result in impaired glymphatic circulation [6]. In histopathological studies of brain tissue specimens of iNPH subjects, astrogliosis has been found, which may induce stiffening of the brain, as well as a loss of perivascular water channels aquaporin-4, which may hamper glymphatic circulation [13]. Accordingly, processes at the glia-vascular interface may be extra-cerebrovascular factors responsible for alterations in pulsatile ICP. Future studies addressing disease processes affecting the extracerebrovascular compartment in iNPH may lay the basis for medical treatment of this dementia disease.

\section{Limitations}

The present approach aiming at differentiation between cerebrovascular and extra-cerebrovascular factors represents a 
simplification, as these factors interact in vivo. Moreover, the cerebrovascular factors incorporate several variables such as cerebral blood flow changes, vascular wall alterations, and cerebrovascular tone-related variations in autoregulation. Likewise, the extra-cerebrovascular factors may involve various alterations in the brain parenchyma and its interaction with CSF. Nevertheless, the study of complex physiological mechanisms requires simplification. In this regard, the differentiation between cerebrovascular and extra-cerebrovascular factors seems one useful approach.

The previous studies examining the moving correlation between ICP and arterial BP waveform amplitudes exclusively utilized peripheral arterial BP measurements, typically from the radial artery $[4,9,10]$. We hypothesized that central aortic BP estimates are better for this purpose. It should be noted, however, that despite the thorough validation, the central aortic BP waveforms used here are indeed estimates. They thereby do provide an additional source of uncertainty in the analysis and are not the perfect proxy for intracranial arterial BP waveforms. The SphygmoCor systems extensive validation study, however, does provide some reassurance of the validity of the estimates [12]. A preliminary study showing a higher similarity between ICP waveforms and central aortic BP waveforms compared to radial arterial BP waveforms further substantiates our observations [18]. Various other epidemiological [21,35] and clinical studies [23, 37] using the SphygmoCor system supports the same conclusion.

\section{Conclusions}

In about $1 / 5 \mathrm{iNPH}$ patients of this study, the intracranial aortic

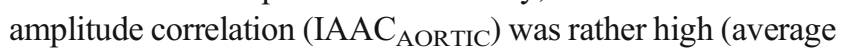
Pearson correlation coefficient $\geq 0.4$ ), suggesting that cerebrovascular factors to some extent may affect the ICP wave amplitudes in a subset of patients. However, in 14/19 (74\%) iNPH patients with elevated ICP wave amplitudes, the intracranial aortic amplitude correlation was low, indicating that the ICP pulse amplitude in most iNPH patients is independent of central vascular excitation, ergo it is modulated by local cerebrospinal physiology. In support of this assumption, the intracranial aortic amplitude correlation was not related to most systemic hemodynamic variables. An exception was found for a subgroup of the patients with high systemic vascular resistance, where there was a correlation.

Acknowledgments The authors thank AtCorMedical Pty Ltd. (West Ryde, Australia) for estimating the central aortic blood pressure waveforms from the radial artery blood pressure waveforms using the SphygmoCor system.

Funding information Open Access funding provided by University of Oslo (incl Oslo University Hospital). Health South-East, Norway, provided financial support in the form of grant 2016032.

\section{Compliance with ethical standards}

Disclaimer The sponsor had no role in the design or conduct of this research.

Conflict of interest PKE has a financial interest in the software company (dPCom AS, Oslo) manufacturing the software (Sensometrics Software) used for analysis of the pressure recordings. KBE and PKE certify that they have no affiliations with or involvement in any organization or entity with any financial interest (such as honoraria; educational grants; participation in speakers' bureaus; membership, employment, consultancies, stock ownership, or other equity interest; and expert testimony or patent-licensing arrangements), or non-financial interest (such as personal or professional relationships, affiliations, knowledge or beliefs) in the subject matter or materials discussed in this manuscript.

Ethical approval The pressure recordings used in this study were retrieved from a study approved by the Regional Ethical Committee of Health Region South-East in Norway (S-07362b) and by Rikshospitalet (07/5870). Informed consent was obtained from all individual participants included in the study. All procedures performed in studies involving human participants were in accordance with the ethical standards of the institutional and/or national research committee (name of institute/committee) and with the 1964 Helsinki declaration and its later amendments or comparable ethical standards.

Open Access This article is licensed under a Creative Commons Attribution 4.0 International License, which permits use, sharing, adaptation, distribution and reproduction in any medium or format, as long as you give appropriate credit to the original author(s) and the source, provide a link to the Creative Commons licence, and indicate if changes were made. The images or other third party material in this article are included in the article's Creative Commons licence, unless indicated otherwise in a credit line to the material. If material is not included in the article's Creative Commons licence and your intended use is not permitted by statutory regulation or exceeds the permitted use, you will need to obtain permission directly from the copyright holder. To view a copy of this licence, visit http://creativecommons.org/licenses/by/4.0/.

\section{References}

1. Aries MJ, Czosnyka M, Budohoski KP, Kolias AG, Radolovich DK, Lavinio A, Pickard JD, Smielewski P (2012) Continuous monitoring of cerebrovascular reactivity using pulse waveform of intracranial pressure. Neurocrit Care 17:67-76

2. Cecconi M, Dawson D, Grounds RM, Rhodes A (2009) Lithium dilution cardiac output measurement in the critically ill patient: determination of precision of the technique. Intensive Care Med 35:498-504

3. Ding F-H, Fan W-X, Zhang R-Y, Zhang Q, Li Y, Wang J-G (2011) Validation of the noninvasive assessment of central blood pressure by the SphygmoCor and Omron devices against the invasive catheter measurement. Am J Hypertens 24:1306-1311

4. Eide PK (2011) Cardiac output in idiopathic normal pressure hydrocephalus: association with arterial blood pressure and intracranial pressure wave amplitudes and outcome of shunt surgery. Fluids Barriers CNS 8:11

5. Eide PK, Pripp AH (2014) Increased prevalence of cardiovascular disease in idiopathic normal pressure hydrocephalus patients compared to a population-based cohort from the HUNT3 survey. Fluids Barriers CNS 11:19 
6. Eide PK, Ringstad G (2019) Delayed clearance of cerebrospinal fluid tracer from entorhinal cortex in idiopathic normal pressure hydrocephalus: a glymphatic magnetic resonance imaging study. J Cereb Blood Flow Metab 39:1355-1368

7. Eide PK, Sorteberg W (2010) Diagnostic intracranial pressure monitoring and surgical management in idiopathic normal pressure hydrocephalus: a 6-year review of 214 patients. Neurosurgery $66: 80$ 91

8. Eide PK, Sorteberg W (2016) Outcome of surgery for idiopathic normal pressure hydrocephalus: role of preoperative static and pulsatile intracranial pressure. World Neurosurg 86:186-193.e181

9. Eide PK, Czosnyka M, Sorteberg W, Pickard JD, Smielewski P (2007) Association between intracranial, arterial pulse pressure amplitudes and cerebral autoregulation in head injury patients. Neurol Res 29:578-582

10. Eide PK, Park EH, Madsen JR (2010) Arterial blood pressure vs intracranial pressure in normal pressure hydrocephalus. Acta Neurol Scand 122:262-269

11. Eide PK, Sorteberg A, Bentsen G, Marthinsen PB, Stubhaug A, Sorteberg W (2012) Pressure-derived versus pressure wave amplitude-derived indices of cerebrovascular pressure reactivity in relation to early clinical state and 12-month outcome following aneurysmal subarachnoid hemorrhage. J Neurosurg 116:961-971

12. Gallagher D, Adji A, O'Rourke MF (2004) Validation of the transfer function technique for generating central from peripheral upper limb pressure waveform. Am J Hypertens 17:1059-1067

13. Hasan-Olive MM, Enger R, Hansson HA, Nagelhus EA, Eide PK (2019) Loss of perivascular aquaporin-4 in idiopathic normal pressure hydrocephalus. Glia 67:91-100

14. Holm S, Eide PK (2009) Impact of sampling rate for time domain analysis of continuous intracranial pressure (ICP) signals. Med Eng Phys 31:601-606

15. Iliff JJ, Wang M, Liao Y, Plogg BA, Peng W, Gundersen GA, Benveniste H, Vates GE, Deane R, Goldman SA, Nagelhus EA, Nedergaard M (2012) A paravascular pathway facilitates CSF flow through the brain parenchyma and the clearance of interstitial solutes, including amyloid beta. Sci Transl Med 4:147ra111

16. Iliff JJ, Wang M, Zeppenfeld DM, Venkataraman A, Plog BA, Liao Y, Deane R, Nedergaard M (2013) Cerebral arterial pulsation drives paravascular CSF-interstitial fluid exchange in the murine brain. $\mathrm{J}$ Neurosci 33:18190-18199

17. Karamanoglu M, O'rourke M, Avolio A, Kelly R (1993) An analysis of the relationship between central aortic and peripheral upper limb pressure waves in man. Eur Heart J 14:160-167

18. Kim MO, Eide PK, O'Rourke MF, Adji A, Avolio AP (2016) Intracranial pressure waveforms are more closely related to central aortic than radial pressure waveforms: implications for pathophysiology and therapy Intracranial Pressure and Brain Monitoring XV. Springer, pp 61-64

19. Kurita T, Morita K, Kato S, Kikura M, Horie M, Ikeda K (1997) Comparison of the accuracy of the lithium dilution technique with the thermodilution technique for measurement of cardiac output. $\mathrm{Br}$ J Anaesth 79:770-775

20. Langesaeter E, Rosseland LA, Stubhaug A (2008) Continuous invasive blood pressure and cardiac output monitoring during cesarean delivery: a randomized, double-blind comparison of low-dose versus high-dose spinal anesthesia with intravenous phenylephrine or placebo infusion. Anesthesiology 109:856-863

21. Li Y, Staessen JA, Li L, Huang Q, Lu L, Wang J (2008) Reference values for the arterial pulse wave in Chinese. Am J Hypertens 21: 668-673

22. Lloyd RA, Stoodley MA, Fletcher DF, Bilston LE (2019) The effects of variation in the arterial pulse waveform on perivascular flow. J Biomech 90:65-70
23. McEniery CM, Yasmin MDB, Munnery M, Wallace SM, Rowe CV, Cockcroft JR, Wilkinson IB (2008) Central pressure: variability and impact of cardiovascular risk factors: the Anglo-Cardiff collaborative trial II. Hypertension 51:1476-1482

24. Mestre H, Tithof J, Du T, Song W, Peng W, Sweeney AM, Olveda G, Thomas JH, Nedergaard M, Kelley DH (2018) Flow of cerebrospinal fluid is driven by arterial pulsations and is reduced in hypertension. Nat Commun 9:4878

25. Pauca AL, O'Rourke MF, Kon ND (2001) Prospective evaluation of a method for estimating ascending aortic pressure from the radial artery pressure waveform. Hypertension 38:932-937

26. Pearse RM, Ikram K, Barry J (2004) Equipment review: an appraisal of the LiDCO plus method of measuring cardiac output. Crit Care 8:190-195

27. Piper IR, Miller JD, Dearden NM, Leggate JR, Robertson I (1990) Systems analysis of cerebrovascular pressure transmission: an observational study in head-injured patients. J Neurosurg 73:871-880

28. Piper IR, Chan KH, Whittle IR, Miller JD (1993) An experimental study of cerebrovascular resistance, pressure transmission, and craniospinal compliance. Neurosurgery 32:805-815 discussion 815-806

29. Pittman J, Bar-Yosef S, SumPing J, Sherwood M, Mark J (2005) Continuous cardiac output monitoring with pulse contour analysis: a comparison with lithium indicator dilution cardiac output measurement. Crit Care Med 33:2015-2021

30. Portnoy HD, Chopp M, Branch C, Shannon MB (1982) Cerebrospinal fluid pulse waveform as an indicator of cerebral autoregulation. J Neurosurg 56:666-678

31. Portnoy HD, Chopp M, Branch C (1983) Hydraulic model of myogenic autoregulation and the cerebrovascular bed: the effects of altering systemic arterial pressure. Neurosurgery 13:482-498

32. Rasmussen MK, Mestre H, Nedergaard M (2018) The glymphatic pathway in neurological disorders. Lancet Neurol 17:1016-1024

33. Relkin N, Marmarou A, Klinge P, Bergsneider M, Black PM (2005) Diagnosing idiopathic normal-pressure hydrocephalus. Neurosurgery 57:S4-S16 discussion ii-v

34. Ringstad G, Vatnehol SAS, Eide PK (2017) Glymphatic MRI in idiopathic normal pressure hydrocephalus. Brain 140:2691-2705

35. Roman MJ, Devereux RB, Kizer JR, Lee ET, Galloway JM, Ali T, Umans JG, Howard BV (2007) Central pressure more strongly relates to vascular disease and outcome than does brachial pressure: the strong heart study. Hypertension 50:197-203

36. Steiner LA, Coles JP, Johnston AJ, Chatfield DA, Smielewski P, Fryer TD, Aigbirhio FI, Clark JC, Pickard JD, Menon DK, Czosnyka M (2003) Assessment of cerebrovascular autoregulation in head-injured patients: a validation study. Stroke 34:2404-2409

37. Williams B, Lacy PS, Thom SM, Cruickshank K, Stanton A, Collier D, Hughes AD, Thurston H, O'Rourke M (2006) Differential impact of blood pressure-lowering drugs on central aortic pressure and clinical outcomes: principal results of the conduit artery function evaluation (CAFE) study. Circulation 113: $1213-1225$

38. Zong W, Heldt T, Moody G, Mark R (2003) An open-source algorithm to detect onset of arterial blood pressure pulses Computers in Cardiology, 2003. IEEE, pp 259-262

39. Zweifel C, Lavinio A, Steiner LA, Radolovich D, Smielewski P, Timofeev I, Hiler M, Balestreri M, Kirkpatrick PJ, Pickard JD, Hutchinson P, Czosnyka M (2008) Continuous monitoring of cerebrovascular pressure reactivity in patients with head injury. Neurosurg Focus 25:E2

Publisher's note Springer Nature remains neutral with regard to jurisdictional claims in published maps and institutional affiliations. 